\title{
Acute Ischemic Stroke Associated With OrthoVisc (Hyaluronic Acid) Injection
}

\author{
Ben-Jiang Ma ${ }^{\mathrm{a}, \mathrm{b}}$, Sampathkumar Shanmugham ${ }^{\mathrm{a}}$
}

\begin{abstract}
Hyaluronic acid (HA) is one of the main components of normal synovial fluid, acting as a shock absorber and a lubricant in the joints. When injected intra-articularly, the viscosupplementation of HA may potentially restore the biomechanical and biochemical functions of normal synovial fluid and may reduce the pain caused by osteoarthritis through anti-inflammatory, anabolic, analgesic, and chondroprotective mechanisms. HA has been approved by FDA as a medical device and has been used in patients with advanced osteoarthritis. Though it is usually safe and well tolerated, some local and systemic reactions can occur in rare cases. Here we report a 53-year-old man with severe osteoarthritis, who developed acute left cerebellar infarct after the injection of HA (OrthoVisc) preceded by symptoms of allergic reaction. Review of literature showed this is to date the first case of possible link between HA and ischemic stroke.
\end{abstract}

Keywords: Hyaluronic acid; OrthoVisc; Ischemic stroke; Systemic reaction

\section{Introduction}

Hyaluronic acid (HA) is a high molecular weight polysaccharidal glycosaminoglycan. HA is one of the main components of normal synovial fluid, acting as a shock absorber and a lubricant in the knee joint. It has been approved by FDA as a medical device to use in patients with advanced osteoarthritis with satisfactory pain relief or control by potentially restoring the biomechanical and biochemical functions of normal synovial fluid. HA is usually safe and well tolerated. However, some local and systemic reactions were reported in rare cases. In this report, we present a case of cerebellar ischemic stroke following intra-articular OrthoVisc (one of the HAs) injection.

Manuscript submitted May 27, 2017, accepted June 15, 2017

aFlorida Neurology, 755 Stirling Center Pl, Lake Mary, FL 32746, USA

${ }^{\mathrm{b}}$ Corresponding Author: Ben-Jiang Ma, Florida Neurology, 755 Stirling Center Pl, Lake Mary, FL 32746, USA. Email: benjiangma@gmail.com

doi: https://doi.org/10.14740/jmc2853w

\section{Case Report}

Recently, a 53-year-old white male patient with past medical history of reportedly well-controlled hypertension, severe osteoarthritis and gastro-esophageal reflux disease (GERD) and previous tobacco use was admitted to the hospital for transient ischemic attack (TIA) symptoms with left-sided weakness and gait imbalance. He had been suffering from severe chronic pain in both knees for years and failed conservative treatment. One day before admission, he had OrthoVisc (high molecular weight HA) injection in both knees at the orthopedic doctor's office. Soon after the procedure, he started having dizziness, weakness and fainting. In the meantime, the patient felt hot and developed macular rashes on his body. In the emergency room (ER), his blood pressure was $153 / 86 \mathrm{~mm} \mathrm{Hg}$. Due to the improvement of the symptoms, and the absence of focal neurologic deficits, he was discharged the same day.

In the early next day morning, the patient woke up with a headache, palpitations and some weakness in the left leg and unsteady gait. He came to the ER again, with initial blood pressure of 144/97 mm Hg, which was better controlled within the normal limits since admission. His symptomatology improved quickly and ultimately resolved. During the neurology consultation, he was completely asymptomatic, and his neurologic exam was essentially within normal limits. His brain magnetic resonance imaging (MRI), however, did show an acute infarct in the left cerebellar vermis as evidenced in diffusionrestriction image (Fig. 1a) and corresponding apparent diffusion coefficient image (Fig. 1b). Computed tomography (CT) angiogram of the head and neck with contrast showed absence of left anterior inferior cerebellar artery (AICA) suggestive of occlusion (Fig. 2) as opposed to the patent right AICA (Fig. 2 , arrow). Rest of the cervical and cerebral vasculatures were without imaging evidence of stenosis, occlusion, or vasculitis.

His labs showed marginally elevated low-density lipoprotein (LDL) of $110 \mathrm{mg} / \mathrm{dL}$ (range $<100 \mathrm{mg} / \mathrm{dL}$ ) and total triglyceride of $164 \mathrm{mg} / \mathrm{dL}$ (range $35-150 \mathrm{mg} / \mathrm{dL}$ ), but otherwise normal complete blood count, normal hemoglobin A1c, normal renal function and liver function. Inflammatory markers including erythrocyte sedimentation rate (ESR, $8 \mathrm{~mm} / \mathrm{h}$, reference range $0-20 \mathrm{~mm} / \mathrm{h}$ ) and high-sensitive C-reactive protein (hsCRP, $1.78 \mathrm{mg} / \mathrm{dL}$, reference range $0-3.0 \mathrm{mg} / \mathrm{dL}$ ) were both within normal limits (Table 1). His 2D echocardiogram showed ejection fraction (EF) of 55-60\% and normal sized left atrium of $3.9 \mathrm{~cm}$ (reference range $3.0-4.0 \mathrm{~cm}$ ) and no evi- 

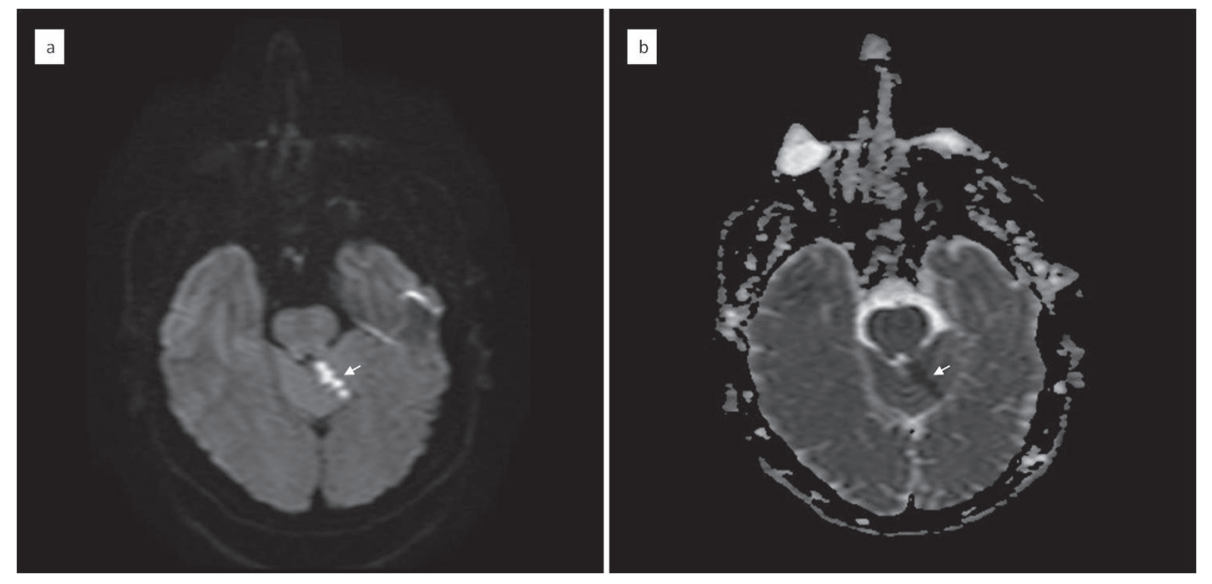

Figure 1. Acute ischemic left cerebellar infarct on MRI (arrows). (a) Diffusion-weighted imaging showing hyperattenuation in the left cerebellum posterior to the pons and the third ventricle. (b) Corresponding apparent diffusion coefficient map showing the hypoattenuation of the same location in the left cerebellum suggestive of acute infarct.

dence of thrombus.

The patient was placed on aspirin, simvastatin along with blood pressure medications and discharged home. He was advised to be cautious of potentially allergic to OrthoVisc or HArelated products. Although no causal effect can be established, the possible association between OrthoVisc and ischemic stroke is of interest.

\section{Discussion}

HA is a high molecular weight polysaccharidal glycosaminoglycan, which is polymerized with repeating disaccharide units of D-glucuronic acid and N-acetyl-D-glucosamine. HA

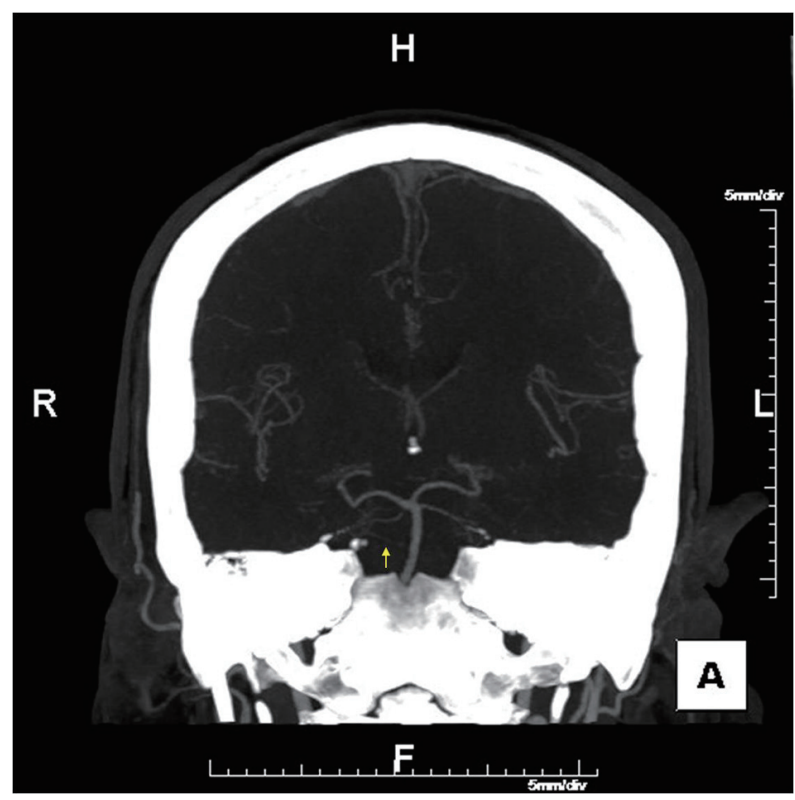

Figure 2. CT angiogram of the head in patient with acute ischemic left cerebellar infarct. Note the absence of left anterior inferior cerebellar artery (AICA) as opposed to the right AICA (arrow). is ubiquitously distributed in the tissues including central nervous system. Being highly viscoelastic, HA is one of the main components of normal synovial fluid, acting as a shock absorber and a lubricant in the knee joint. In patient with osteoarthritis, the elevated levels of free radicals, inflammatory cytokines, and enzymes in the synovial fluid impair the function of HA and contribute to the disease progression. Physiologically, when injected intra-articularly, the viscosupplementation of HA may potentially restore the biomechanical and biochemical functions of normal synovial fluid and may reduce the pain caused by osteoarthritis through anti-inflammatory, anabolic, analgesic, and chondroprotective mechanisms [1].

Study by Petrella (2005) involving nearly 900 patients showed that the long-term symptom control (6 months) with HA injection was highly satisfactory [2]. A 2006 Conchrane systemic review and meta-analysis of 76 trials noted the positive efficacy of HA injection is non-inferior to non-steroidal anti-inflammatory drugs (NSAIDs) and longer-term benefits being similar or superior to intra-articular (IA) corticosteroids [3]. A recent systemic review in 2015 by the Center for Medicare and Medicaid Services (CMS) confirmed a small but statistically significant improvement in the articular function with HA injection [4]. Although no conclusion was drawn on the delay or avoidance of total knee replacement for patients with severe osteoarthritis, one study did show prolonged benefit for symptom relief and improvement of function up to 2 years with HA viscosupplementation. This beneficial effect was more pronounced with combined low and high molecular weight HA injections [5].

Thanks to its proven benefit, in the past decade, the intra-articular HA viscosupplementation has gained increasing popularity as an alternative treatment option for non-operative management of osteoarthritis [6]. HA-based viscosupplements have been approved by FDA as medical device instead of medicine, due to that the level of efficacy demonstrated is less than might have been required for approval as a drug [7,8]. Currently, there are several commercially available HA-based viscosupplement products, which are generally categorized in two types: the HA (hyaluronan)-based and the sodium hya- 
Table 1. Patient's Pertinent Laboratory Data

\begin{tabular}{|c|c|c|c|}
\hline Labs & Value & Units & Reference range \\
\hline \multicolumn{4}{|l|}{ Lipid panel } \\
\hline Total triglyceride & 164 & $\mathrm{mg} / \mathrm{dL}$ & $30-150$ \\
\hline Total cholesterol & 189 & $\mathrm{mg} / \mathrm{dL}$ & $<199$ \\
\hline LDL cholesterol & 110 & $\mathrm{mg} / \mathrm{dL}$ & $<100$ \\
\hline HDL cholesterol & 46 & $\mathrm{mg} / \mathrm{dL}$ & $>41$ \\
\hline VLDL cholesterol & 33 & $\mathrm{mg} / \mathrm{dL}$ & $<30$ \\
\hline Total cholesterol/HDL & 4.1 & & \\
\hline LDL/HDL & 2.4 & & \\
\hline \multicolumn{4}{|l|}{ Chemistry } \\
\hline Sodium & 139 & $\mathrm{mmol} / \mathrm{L}$ & $135-145$ \\
\hline Potassium & 4.1 & $\mathrm{mmol} / \mathrm{L}$ & $3.4-5.5$ \\
\hline Chloride & 102 & $\mathrm{mmol} / \mathrm{L}$ & $95-105$ \\
\hline $\mathrm{CO}_{2}$ & 24 & $\mathrm{mmol} / \mathrm{L}$ & $20-30$ \\
\hline BUN & 14 & $\mathrm{mg} / \mathrm{dL}$ & $7-22$ \\
\hline Creatinine & 1.0 & $\mathrm{mg} / \mathrm{dL}$ & $0.44-1.03$ \\
\hline Fasting blood glucose & 101 & $\mathrm{mg} / \mathrm{dL}$ & $70-99$ \\
\hline Calcium & 9.1 & $\mathrm{mg} / \mathrm{dL}$ & $8.4-10.8$ \\
\hline Magnesium & 2.1 & $\mathrm{mg} / \mathrm{dL}$ & $1.7-2.3$ \\
\hline $\mathrm{HbA1C}$ & 5.2 & $\%$ & $3.5-5.5$ \\
\hline \multicolumn{4}{|l|}{ Liver function } \\
\hline Total protein & 6.7 & $\mathrm{~g} / \mathrm{dL}$ & $6.4-8.2$ \\
\hline Albumin & 4.3 & $\mathrm{~g} / \mathrm{dL}$ & $3.4-5.0$ \\
\hline ALT & 20 & units & $12-78$ \\
\hline AST & 15 & units & $15-37$ \\
\hline Total bilirubin & 0.8 & $\mathrm{mg} / \mathrm{dL}$ & \\
\hline Alkaline phosphatase & 39 & units & $45-117$ \\
\hline \multicolumn{4}{|l|}{ Inflammation markers } \\
\hline HsCRP & 1.78 & $\mathrm{mg} / \mathrm{dL}$ & $<3.5$ \\
\hline ESR & 8 & $\mathrm{~mm} / \mathrm{h}$ & $0-20$ \\
\hline \multicolumn{4}{|l|}{ Complete blood count } \\
\hline WBC & 7.6 & $10^{3} / \mu \mathrm{L}$ & $4.0-11.0$ \\
\hline $\mathrm{RBC}$ & 4.85 & $10^{6} / \mu \mathrm{L}$ & $3.8-5.2$ \\
\hline Hemoglobin & 15.4 & $\mathrm{~g} / \mathrm{dL}$ & $12.0-15.4$ \\
\hline Hematocrit & 45.6 & $\%$ & $35-45$ \\
\hline RDW & 13 & $\%$ & $11.5-14.5$ \\
\hline Platelet & 282 & $10^{3} / \mu \mathrm{L}$ & $150-400$ \\
\hline \multicolumn{4}{|l|}{ Coagulation } \\
\hline Prothrombin time & 12 & $\mathrm{~s}$ & $9.0-11.6$ \\
\hline INR & 0.89 & & $2.0-3.0$ \\
\hline PTT & 23.8 & $\mathrm{~s}$ & $24.0-32.0$ \\
\hline
\end{tabular}

LDL: low-density lipoprotein; HDL: high-density lipoprotein; VLDL: very low-density lipoprotein; BUN: blood urea nitrogen; ALT: alanine aminotransferase; AST: aspartate aminotransferase; hsCRP: high-sensitive C-reactive protein; ESR: erythrocyte sedimentation rate; WBC: white blood cell; RBC: red blood cell; RDW: red cell distribution width; PT: prothrombin time; INR: international normalized ratio; PTT: partial thromboplastin time. 
luronate-based high molecular weight polymers. The former include OrthoVisc, Monovisc and Hylan G-F 20 (Synvisc, a cross-linked preparation of high molecular weight hyaluronan). OrthoVisc has a molecular weight ranging 1.0 - 2.9 million Daltons. It was initially developed by Anika Therapeutics, Inc. and approved by FDA as medical device in 2003 [8].

In general, the injection of HA is considered safe. The majority reported adverse reactions are local and self-limited, including edema, erythema, increased pain and stiffness of the knee joint which usually resolved quickly $[2,5,6]$. Both the 2006 Cochrane review and 2015 CMS review found few no statistically significant reactions than the placebo with hyaluronan injections. Some local reactions appeared to be technique-related events, such as infection (septic arthritis), nerve injury (saphenous nerve), etc. [3, 4].

However, several studies have found more serious adverse effects and complications with HA injection. One report showed that intra-articular injection of Hylan G-F 20 was associated with isolated or recurrent acute calcium pyrophosphate dehydrate (CPPD) arthritis (pseudogout arthritis) [9]. These authors proposed that the interaction between fragmented HA and its intracellular receptor CD44 might be one of the mechanisms for the recruitment of white blood cells that produce pro-inflammatory factors [10].

In addition to the localized adverse reactions with HA injections, cases of systemic adverse reactions were also reported. In 2001, Martens reported a patient who developed severe symmetric pseudoseptic arthritis (the synovial fluid was negative for bacteria and CPPD) in bilateral knees following the first injection of Hylan G-F 20 in the second course of treatment [11]. Rees et al reported a patient who received repeated OrthoVisc injection developed fever and inflammation (elevated CRP and ESR), leukocytosis and eosinophilia in the absence of septic or gout/pseudogout arthritis, although the patient did have large intra-articular effusion [12]. Calvo et al reported a case of systemic reaction to HA injection manifested with erythema multiform [13]. These studies suggested that the pre-sensitization by HA-based products was the trigger of this exaggerated severe acute inflammatory reactions (SAIR) and some individuals might be more susceptible than others.

Further study by Bucher et al, using the different hyaluronate products (HA and sodium hyaluronate) to immunize rabbits found that positive antibodies against chicken protein were developed by the rabbits immunized with repeated HA but not sodium hyaluronate (Hyalgan) [14]. This study suggested that the immunogenic properties of different hyaluronate products differ from each other and the development of humoral response to HA required repeated immunization (sensitization), which explained the cases previously reported by Martens with SAIR [11]. The anti-chicken protein antibodies could be elicited by the more immunogenic degraded small fragment HA [14]. Further clinical trials led by Brown et al with head-to-head comparison of the safety between Hylan G-F 20 and sodium hyaluronate (Hyalgan) showed that 6/29 (20.6\%) Hylan-treated patients developed SAIR while none of the Hyalgan-treated patients $(n=25)$ had such complications [15]. Therefore, the HA-based viscosupplement products (Synvisc, OrthoVisc) may carry safety risks with uncertain clinical consequences, which may be more serious in patients who are allergic to egg or egg products. As endogenous HA is widely distributed in all the tissues in human body, including the brain, the impact of the immune responses induced by the exogenous HA may be one of the key components for the development of adverse reactions.

A recently published study by Tang et al showed that in both ischemic and hemorrhagic stroke patients, the serum HA levels were significantly elevated. In hemorrhagic stroke patients, the HA level (measured within $48 \mathrm{~h}$ from the onset of symptoms) greater than $500 \mathrm{ng} / \mathrm{mL}$ was associated with the worse outcome of recovery. However, in patients with brain infarction, an inverted U-shaped pattern was identified between the HA level and the outcome of recovery at 3 months [16]. The HA was considered as one of the pro-inflammatory factors induced by acute stroke through activation hyaluronidase, which degrades the high molecular weight HA polymers to heterogenous smaller fragmented polysaccharide HAs. These degraded HAs are the ligand of Toll-like receptors, which trigger the extensive immune responses that may result in the secondary injury in the central nervous system to the adjacent tissues surrounding the initial stroke insult (penumbra), which, with the balancing cell repair, ultimately determine the extent of brain damage and degree of recovery [16].

In our case, the patient had left cerebellar infarct due to the occlusion of left AICA following intra-articular HA (OrthoVisc) injection. Prior to the stroke, he had systemic allergic reactions after the OrthoVisc injection and elevated blood pressure, which is a risk factor for developing lacunar infarct. Although the causal effect between the exogenous HA-induced inflammatory response and the acute stroke cannot be established due to the rarity of the cases, the association between the two consecutive events deserves further investigation.

\section{Grants}

None.

\section{Disclosure}

The authors have nothing to disclose.

\section{References}

1. Evaniew N, Simunovic N, Karlsson J. Cochrane in CORR(R): Viscosupplementation for the treatment of osteoarthritis of the knee. Clin Orthop Relat Res. 2014;472(7):2028-2034.

2. Petrella RJ. Hyaluronic acid for the treatment of knee osteoarthritis: long-term outcomes from a naturalistic primary care experience. Am J Phys Med Rehabil. 2005;84(4):278-283; quiz 284, 293.

3. Bellamy N, Campbell J, Robinson V, Gee T, Bourne R, Wells G. Viscosupplementation for the treatment of osteoarthritis of the knee. Cochrane Database Syst Rev. 
2006;2:CD005321.

4. Newberry SJ, Fitzgerald JD, Maglione MA, O'Hanlon CE, Booth M, Motala A, Timmer M, Shanman R, Shekelle PG. Systematic review for effectiveness of hyaluronic acid in the treatment of severe Degenerative Joint Disease (DJD) of the knee [Internet]. Rockville (MD): Agency for Healthcare Research and Quality (US); 2015.

5. Petrella RJ, Decaria J, Petrella M. Long term efficacy and safety of a combined low and high molecular weight hyaluronic acid in the treatment of osteoarthritis of the knee. Rheumatology Reports. 2001;3(1):e4.

6. Watterson JR, Esdaile JM. Viscosupplementation: therapeutic mechanisms and clinical potential in osteoarthritis of the knee. J Am Acad Orthop Surg. 2000;8(5):277-284.

7. http://www.accessdata.fda.gov/cdrh_docs/pdf3/P0300 19b.pdf.

8. http://www.accessdata.fda.gov/scripts/cdrh/cfdocs/cftop$\mathrm{ic} / \mathrm{pma} / \mathrm{pma} . \mathrm{cfm}$ ?num=p03001.

9. Disla E, Infante R, Fahmy A, Karten I, Cuppari GG. Recurrent acute calcium pyrophosphate dihydrate arthritis following intraarticular hyaluronate injection. Arthritis Rheum. 1999;42(6):1302-1303.

10. Punzi L, Pianon M, Piero SG, Todesco S. Pseudogout and intraarticular hyaluronate injections: comment on the article by Disla et al. Arthritis Rheum. 2000;43(7):1660-1661 .
11. Martens PB. Bilateral symmetric inflammatory reaction to hylan G-F 20 injection. Arthritis Rheum. 2001;44(4):978979.

12. Rees JD, Wojtulewski JA. Systemic reaction to viscosupplementation for knee osteoarthritis. Rheumatology (Oxford). 2001;40(12):1425-1426.

13. Calvo M, Tornero P, De Barrio M, Minguez G, Infante S, Herrero T, Baeza ML. Erytema multiform due to hyaluronic acid (go-on). J Investig Allergol Clin Immunol. 2007;17(2):127-128.

14. Bucher W, Otto T, Hamburger MI. Differentiation of hyaluronate products by qualitative differences in their immunogenicity in rabbits: possible mechanism for productspecific severe adverse reactions? Comment on the article by Martens. Arthritis Rheum. 2002;46(9):2543-2544; author reply 2544.

15. Brown DJ, Wood EV, Hannah HM, Rao VS, Teanby D. Prospective comparison of sodium hyaluronate and hylan G-F 20 in a clinical practice: comment on the concise communication by Martens. Arthritis Rheum. 2004;50(5):1697-1698; author reply 1698.

16. Tang SC, Yeh SJ, Tsai LK, Hu CJ, Lien LM, Peng GS, Yang WS, et al. Association between plasma levels of hyaluronic acid and functional outcome in acute stroke patients. J Neuroinflammation. 2014;11:101. 